\title{
Los videojuegos en la educación: Beneficios y perjuicios
}

\author{
Videogames in Education: Benefits and Harms \\ Os vídeojogos na educação: benefícios e prejuízos
}

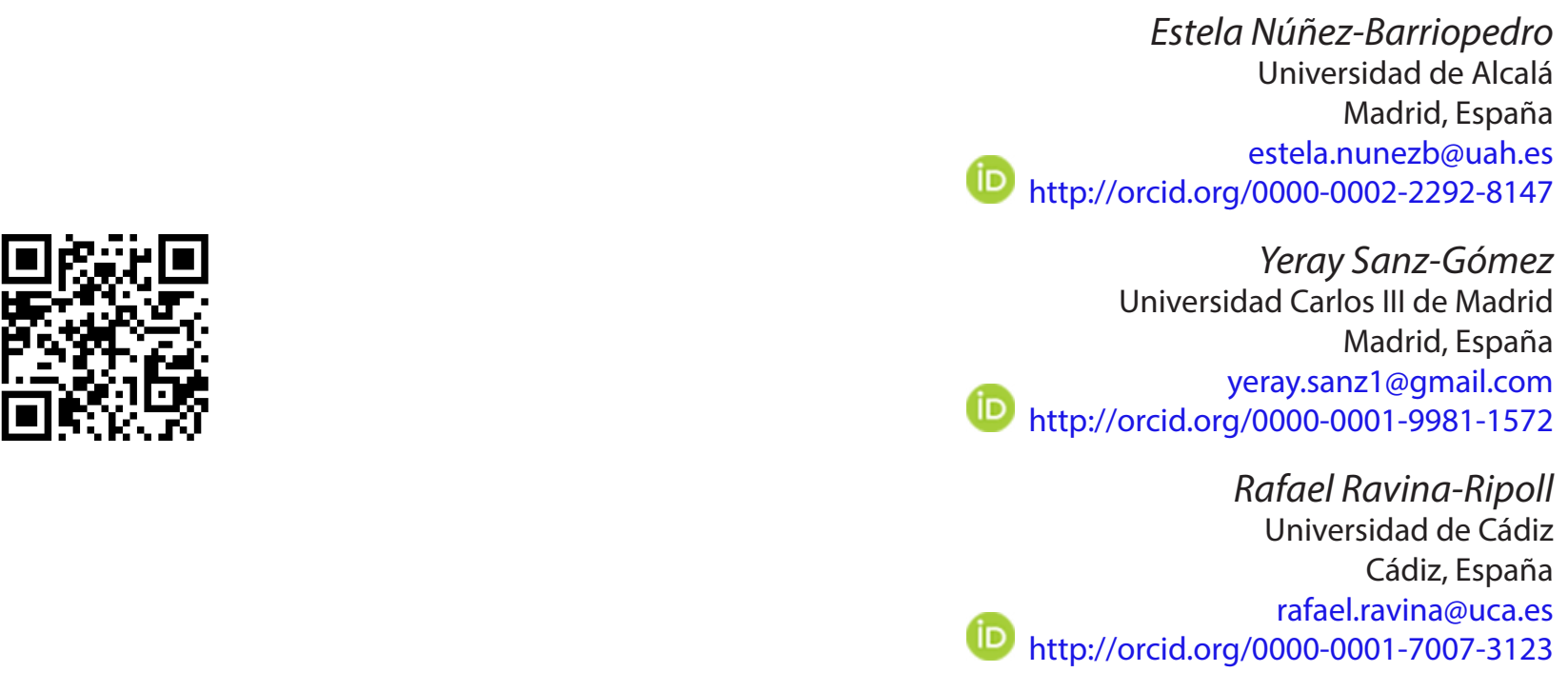

Recibido • Received • Recebido: $06 / 11 / 2018$

Corregido • Revised • Revisado: 27 / 01 / 2020

Aceptado • Accepted • Aprovado: 01 / 04 / 2020

\begin{abstract}
Resumen: Desde el ámbito de la educación, se plantea un cambio de paradigma hacia un proceso enseñanza-aprendizaje más innovador. Concretamente, incluyendo en las aulas videojuegos como recurso docente con la finalidad de conseguir una educación de calidad más entretenida, eficaz y motivadora para el estudiantado. El objetivo principal de esta investigación es analizar si existen diferencias significativas entre las personas consumidoras de videojuegos sobre los beneficios o perjuicios que estos aportan especialmente en educación o si, por el contrario, existe una opinión generalizada positiva o negativa sobre la influencia de los videojuegos en el comportamiento de las personas usuarias. Para el análisis de las perspectivas de los videojuegos se ha llevado a cabo un estudio exploratorio, seguido de un estudio correlacional mediante encuesta donde se ha empleado el análisis clúster y el análisis factorial de correspondencia. En este estudio participaron 400 personas del conjunto nacional de España con edades comprendidas entre los 11 y 64 años. Entre los resultados obtenidos se destaca la creación de varios grupos de personas con diferencias significativas en sus actitudes sobre los videojuegos, en unos casos muy positivas y en otros, algo más conservadoras. Estos resultados permiten concluir que la opinión de las personas consumidoras acerca de cómo los videojuegos pueden influir en el desarrollo personal son más positivas, abriendo las puertas a un uso en la educación y en otros sectores.
\end{abstract}

Palabras claves: Aprendizaje interactivo; competencias; desarrollo cognitivo; estrategias educativas; motivación; videojuegos. 
http://doi.org/10.15359/ree.24-2.12

ROR: https://ror.org/01t466c14 Universidad Nacional, Costa Rica

http://www.una.ac.cr/educare

educare@una.cr

\begin{abstract}
In education, a paradigm is shifting to a more innovative teaching-learning process. Specifically, this paradigm focuses on including video games in classrooms as a teaching resource intended to obtain a more entertaining, efficient, and pleasant quality education for students. The proposed research aims to analyze whether there are significant differences among users of video games on the benefits or harms they bring, especially in education, or there is a positive or negative general opinion, as other authors noted, on the influence of video games on users'behavior. To analyze the viewpoints on video games, first, we do an exploratory study through the literature. Then, we do a correlational survey study where Cluster Analysis and Multiple Correspondence Analysis are used. In this study, we took 400 Spanish consumers aged between 11 and 64 years without discrimination, regardless of sex. Among the results obtained, we can emphasize the creation of several groups with significant differences in their points of view, in some cases, very positive and in others more conservative. These results lead to conclude that the users' opinions about how video games can affect their self-growth are being more positive, opening doors for using video games in education and other sectors.
\end{abstract}

Keywords: interactive learning; skills; cognitive development; education strategies; motivation; video games.

Resumo: No âmbito da educação propõe-se uma mudança de paradigma para um processo de ensino-aprendizagem mais inovador, concretamente, incluindo videojogos nas aulas, como recurso didático, com a finalidade de alcançar uma educação de qualidade, mais atraente, eficaz e motivadora para os estudantes. O objetivo principal desta pesquisa é analisar se existem diferenças significativas entre os consumidores de videojogos sobre os benefícios ou prejuízos que isto implica, especialmente na educação ou se, pelo contrário, existe uma opinião positiva ou negativa generalizada como indicam outros autores, sobre a influência dos videojogos no comportamento dos usuários. Para a análise das perspectivas dos videojogos fez-se um estudo exploratório, seguido de um estudo correlacional, mediante questionário em que se empregou a análise de cluster e a análise fatorial de correspondência. Participaram do estudo 400 pessoas do âmbito nacional da Espanha, com idades compreendidas entre 11 e 64 anos. Entre os resultados obtidos se destaca a criação de vários grupos com diferenças significativas em suas atitudes sobre os videojogos, em alguns casos muito positivas e em outros, mais conservadoras. Estes resultados permitem concluir que a opinião dos consumidores acerca de como os videojogos podem influir no desenvolvimento pessoal tem sido mais positivo, abrindo portas para o uso na educação e em outros setores.

Palavras-chaves: Aprendizagem interativa; competências; desenvolvimento cognitivo; estratégias educativas; motivação; videojogos.

\title{
Introducción
}

El estudio del impacto de los videojuegos es una temática de especial interés, no solo desde la perspectiva de las personas consumidoras, las empresas productoras y las administraciones públicas; sino también por ser los videojuegos un agente socializador que posee una gran influencia en los valores culturales que van adquiriendo nuestra niñez y adolescentes. Resulta 
fundamental destacar que los videojuegos influyen sobre aquella parte de la población que la consume, por ello se están llevando estudios sobre las implicaciones cognitivas que conllevan el videojuego y cómo influyen en la educación (Gros, 2000; Marín Díaz y García Fernández, 2005).

Por una parte, están los partidarios que ofrecen una vista más positiva, referente a los argumentos de aquellos videojuegos donde se presenta una simulación de la realidad. Esto le permite a la niñez descubrir, de una manera menos abstracta, nuevos elementos que le ayudarán a formarse en su proceso de aprendizaje. Sin embargo, lo más destacable es que las personas adolescentes los descubren con los esfuerzos cognitivos que ya conocen o les son familiares (Crescenzi Lanna y Grané Oró, 2016; Gramigna y González-Faraco, 2009). Otra de las ventajas que brindan los videojuegos es que permiten aprender mediante la experiencia de mundos online y la interacción con entes virtuales basados en complejos algoritmos (Hernández y Cano, 2016; Martínez, 2019).

Por otra parte, están aquellos sujetos que ven más limitaciones de aprendizaje sobre aquellos que juegan a los videojuegos e incluso quienes los consideran un impacto negativo (Serna-Rodrigo, 2020). En esta línea, el punto más negativo del consumo de videojuegos es que poseen tanto violencia implícita como explícita, lo cual genera agresividad en quienes los juegan, tanto durante las sesiones de juego como posteriormente (Téllez Quirós, 2019). Otros grupos critican que en los videojuegos se reproducen estereotipos sexistas (Díez Gutiérrez, 2007; Ricoy y Ameneiros, 2016). También existe un creciente temor entre un elevado porcentaje de la población-especialmente padres, madres y educadores- que piensan que los videojuegos anulan la personalidad de las personas adolescentes. Esto no les ayuda a diferenciar entre la realidad y la ficción. Sin embargo, hay numerosos estudios, como los realizados por Greenfield y Cocking (1996) entre otros, que desmontan dichos mitos.

La revisión constante de los beneficios que aportan los videojuegos ofrece la posibilidad de crear nuevas tecnologías más orientadas a otros ámbitos diferentes al sector del ocio. Pérez (2011) demuestra una clara aportación de la realidad virtual a sectores como la medicina por medio de simuladores fundamentados en videojuegos, Asimismo, afirma que son herramientas muy útiles para el diagnóstico y el tratamiento de problemas neuronales y fobias. Por otro lado, existen Mañas Pérez y Roig-Vila (2019) revelan las posibilidades infinitas que posee la realidad virtual dentro del ámbito educativo. Tal hecho viene motivado básicamente por la creación de espacios virtuales donde las personas consumidoras pueden desarrollarse de una manera más social y entretenida.

A parte de la implicación social y educativa que pueden tener los videojuegos, también tienen una importante carga económica en los países. La Asociación Española de Empresas Productoras y Desarrolladoras de Videojuegos (DEV), en su informe del 2015, recoge las previsiones económicas de tres grandes empresas: Newzoo, PWC, y DFC Intelligence. Acorde 
http://doi.org/10.15359/ree.24-2.12

ROR: https://ror.org/01t466c14 Universidad Nacional, Costa Rica

http://www.una.ac.cr/educare

educare@una.cr

con los informes económicos de Newzoo, el mercado global de los videojuegos en el año 2014 fue de 83600 millones de dólares y prevé que en el 2018 alcance unas cifras de 113300 millones de dólares. PWC prevé una estimación más conservadora de 93180 millones para el 2019. DFC Intelligence prevé también unas cifras similares, aunque matiza que solo pasaría de los 100.000 millones en 2019 (DEV, 2015). Estos datos económicos, junto con el crecimiento acelerado, en los últimos años, del público consumidor de videojuegos, en España pasan del 22,5 \% (Interactive Software Federation of Europe [ISFE], 2019) al 36 \% (Asociación Española de Videojuegos [AEVI], 2009) de la población en apenas 6 años. Esto hace del sector del videojuego un buen atractivo para la innovación y el desarrollo.

Existen publicaciones unilaterales que versan tanto sobre los beneficios que pueden tener los videojuegos en el desarrollo de las personas (Capel Masip et ál., 2017; Revuelta y Guerra, 2012). Sin embargo, no se encuentran un número importante de producciones académicas que enfrenten ambas consecuencias. Una de las novedades del presente estudio radica en la consideración bilateral de analizar las percepciones de los individuos acerca de si un videojuego aporta beneficios o contribuye negativamente a su bienestar individual (Núñez-Barriopedro et ál., 2019).

El presente artículo emana de una investigación más amplia que corresponde al Trabajo Fin de Grado de (Sanz-Gómez, 2017) y ha sido codirigido con el resto de personas coautoras de este trabajo.

\section{Marco conceptual}

En la revisión de la bibliografía de este trabajo se expone cómo pueden influir los videojuegos sobre las personas (Halbrook et ál., 2019; Teng et. al, 2020). Unos estudios son partidarios de que al emplear más tiempo en los videojuegos decrece el uso del tiempo en otros tipos de actividades culturales como leer o ver películas (Egenfeldt-Nielsen, 2019). Otros echan la culpa a los videojuegos de las posibles acciones agresivas esporádicas de algunas personas que suelen hacer uso de este entretenimiento (Kühn et. al, 2019). Y, por último, están quienes afirman que, debido al realismo de los videojuegos, la pared entre lo que es ficción y realidad es tan fina que muchas veces los niños o niñas no saben distinguir donde se encuentran (GonzálezVázquez e Igartua Perosanz, 2020; Gramigna y González-Faraco 2009). Además, pueden existir indicios de que una de las causas del bullying en las aulas escolares se pueda deber al uso de violencia y escenas donde se aprecia a los sujetos perdedores en algunos juegos de fama reconocida como el conocido Mortal Kombat (Díez Gutiérrez, 2007; Díez Gutiérrez et ál., 2001).

Ahora bien, estas ideas un tanto retrógradas han ido evolucionando, pues se ha descubierto que los videojuegos poseen componentes que permiten el desarrollo cognitivo de los individuos al usar las teorías de la motivación y del aprendizaje (Etxeberría, 2008; ValderramaRamos, 2012).

4 
http://doi.org/10.15359/ree.24-2.12

ROR: https://ror.org/01t466c14 Universidad Nacional, Costa Rica http://www.una.ac.cr/educare

educare@una.cr

Tanto la teoría de la motivación como la del aprendizaje poseen elementos comunes con los videojuegos, e influyen en el desarrollo individual de quien los juega.

La teoría del aprendizaje tiene como punto clave que "la capacidad humana de emplear símbolos permite representar los fenómenos, analizarsu experiencia consciente, planear, imaginar y actuar de manera previsora" (Etxeberría Balerdi, 2008, p. 9). La relación que presenta con los videojuegos es que, en algunos casos, como, por ejemplo, en la saga Uncharted, desarrollada por Naughty Dog en 2007, se debe hacer uso de dicha capacidad para poder solucionar diferentes puzzles que permiten avanzar en el juego, es decir, se promueve una interacción por parte del sujeto jugador (Rojas, 2008). Incluso Mainer (2006) defiende que el público usuario adquiere cinco aprendizajes significativos como son la destreza motora, comprensión y asimilación de información verbal, destrezas intelectuales, actitudes como la ética moral y estrategias cognoscitivas, ya que potencian la atención y la concentración. La misma autora indica que ayuda a fomentar valores como el respeto a la autoridad, la equidad, la justicia debido a las normas y la colaboración y el deber de auxilio debido a su componente social.

Respecto a la teoría de la motivación, uno de sus pilares es que una tarea no solo tenga el suficiente atractivo o motivación para promover el aprendizaje, sino además deben presentar unos refuerzos que ayuden a cumplimentarla. Un ejemplo de esto sería la posibilidad de alcanzar el nivel máximo en un videojuego y las recompensas que te dan al pasar a ciertos niveles (Sedeño, 2010).

Marquès (2000) da otra visión de los videojuegos, asociando cada tipo de juego con diversas habilidades que se van aprendiendo. Especialmente, les atribuye la mejora de la orientación espacial y el desarrollo psicomotor.

Aparte del uso de las teorías para explicar los beneficios de los videojuegos, presentan aspectos potencialmente beneficiosos para la juventud en su desarrollo. Entre ellos se pueden destacar los aspectos cognitivos, las destrezas y habilidades, los aspectos socializadores y la alfabetización digital (Cole y Griffiths, 2007; Montes-González et ál., 2018).

Los videojuegos pueden ser usados como una herramienta para potenciar la capacidad narrativa de la niñez o para cambiar su estado de ánimo. Russoniello et ál. (2009) demuestran que jugar a videojuegos de puzzles ayuda a cambiar el estado del ánimo, provocando cierta relajación.

González et ál. (2007) identifican un factor bastante beneficioso por parte de los videojuegos para las personas necesitadas de una educación especial, ya que se pueden adaptar a sus dificultades y permiten una mayor comprensión de lo que se enseña. Existen autores y autoras que afirman de la existencia de ciertos juegos que pueden ayudar con temas relativos a la adquisición de hábitos saludables (Marín Díaz y García Fernández, 2005; PinelMartínez et. al., 2016). 
http://doi.org/10.15359/ree.24-2.12

ROR: https://ror.org/01t466c14 Universidad Nacional, Costa Rica

http://www.una.ac.cr/educare

educare@una.cr

Otros enfoques que se pueden dar a los videojuegos como factor educativo son los siguientes:

- Aprendizaje inmersivo. El jugar te permite estar expuesto a una combinación de vivencias, experiencias y toma de decisiones, lo que permite al individuo aprender con base en la unión de dichas características (Castellón y Jaramillo, 2012; Sedeño, 2010).

- El desarrollo de soft- skills. Tradicionalmente la educación estaba enfocada fundamentalmente en la adquisición de contenidos. Actualmente, el paradigma de la educación ha evolucionado a una enseñanza-aprendizaje por competencias. Mediante el uso de los videojuegos puedes desarrollar diferentes tipos de competencias como pueden ser la resolución de problemas y superación de retos (López Gómez y Rodríguez Rodríguez, 2016; Revuelta y Guerra, 2012).

- El aprendizaje complejo. Los videojuegos tienen la capacidad de atraer la atención de la niñez y potenciar su curiosidad mientras están aprendiendo. Esto se conoce como "edu-tainment" (Griffiths, 2002; Jarvin, 2015).

\section{Metodología de la investigación}

Para el desarrollo de esta investigación se ha efectuado la siguiente metodología. Primero, se realizó un estudio exploratorio con revisión bibliográfica, para recoger las principales aportaciones desde los 90 hasta la actualidad, sobre los beneficios o perjuicios del consumo de videojuegos y la educación, seguido de un estudio correlacional mediante encuesta con planteamiento de hipótesis. Posteriormente, se describe el tipo de muestra usada en el análisis. En tercer lugar, se explican las técnicas estadísticas multivariantes que se llevan a cabo con los datos extraídos de la muestra.

\section{Planteamiento de la hipótesis}

Hipótesis 1. El público consumidor de videojuegos se diferencia de modo significativo en función de las percepciones positivas y negativas de cómo influyen estos en la educación.

El impacto de los videojuegos en el día a día de quienes los consumen es un tema que presenta mucha controversia. Los posibles efectos negativos aún presentes de forma residual en la mentalidad de muchas personas consumidoras vienen reforzados por la aparición de noticias en los medios audiovisuales que achacan el uso de los videojuegos con situaciones delictivas o comportamientos impropios (Díez Gutiérrez, 2007; Díez Gutiérrez et ál., 2001).

El objetivo principal de la investigación es analizar si existen diferencias significativas entre los sujetos consumidores de videojuegos sobre los beneficios o perjuicios que estos aportan, especialmente en educación o si, por el contrario, existe una opinión generalizada positiva o negativa, tal como apuntan otras personas autoras sobre la influencia de los videojuegos en el comportamiento de su público consumidor. 
http://doi.org/10.15359/ree.24-2.12

ROR: https://ror.org/01t466c14 Universidad Nacional, Costa Rica

\section{Diseño de la investigación}

Para llevar a cabo esta investigación empírica se han utilizado tanto datos primarios como secundarios. El trabajo de campo mediante encuesta ha sido dirigido a un público objetivo entre los 11 y los 64 años. El cuestionario se ha diseñado, expresamente, para desarrollar este trabajo y se administró entre febrero y abril del 2018.

Con el tamaño de la muestra obtenida (400 personas), el error de muestreo se sitúa, para un intervalo de confianza del 95,5 \%, en el +/- 5. La población objeto de estudio son los habitantes del conjunto nacional de España y el método de muestreo ha sido estratificado.

Los rangos de edad escogidos para la realización del estudio son: de 11-14 años, de 15-24 años, de 25-34 años, de 35-44 años y 45-64 años. Los rangos usados han sido definidos de este modo, para poder compararlos posteriormente con la bibliografía (ISFE, 2019). Se han escogido diferentes rangos de edad para poder observar la penetración de los videojuegos en diferentes edades y poder ver, así, que influencias tienen.

Los estratos de la muestra se han definido atendiendo a la revisión bibliográfica en distintos países como son Francia, Alemania y Reino Unido así la muestra queda distribuida en España en las proporciones expuestas en la Tabla 1.

Tabla 1: Distribución de los grupos consumidores de videojuegos de Francia, Alemania, Reino Unido con base en la edad y el sexo

\begin{tabular}{lcccc}
\hline & Francia & Alemania & Reino unido & España (muestra) \\
\hline 11-14 años & $89 \%$ & $86 \%$ & $75 \%$ & $85 \%$ \\
$15-24$ años & $82 \%$ & $83 \%$ & $59 \%$ & $75 \%$ \\
$25-34$ años & $69 \%$ & $62 \%$ & $39 \%$ & $60 \%$ \\
$35-44$ años & $65 \%$ & $61 \%$ & $38 \%$ & $13 \%$ \\
$45-64$ años & $37 \%$ & $34 \%$ & $20 \%$ & $13 \%$ \\
Mujer & $51 \%$ & $48 \%$ & $43 \%$ & $40 \%$ \\
Hombre & $49 \%$ & $52 \%$ & $57 \%$ & $60 \%$ \\
\hline
\end{tabular}

Nota: Elaboración propia a partir de los datos de ISFE (2019).

Asimismo, los rangos entre los 15-24 y 25-34 años son los que más tiempo dedican al mundo del videojuego (Interactive Advertising Bureau Spain [IAB], 2017).

\section{Variables de la invesigación}

Las variables que se han definido en el cuestionario (ver tabla 2) recogen información, por un lado, sobre las percepciones que los grupos consumidores poseen acerca de los beneficios 
http://doi.org/10.15359/ree.24-2.12

ROR: https://ror.org/01t466c14 Universidad Nacional, Costa Rica

http://www.una.ac.cr/educare

educare@una.cr

o daños que pueden hacer los videojuegos (variable métrica medida con escala de Likert de 5 puntos), por otrolado, variables categóricas que recogen información sobre el perfil demográfico (edad, sexo) y su ocupación actual.

Tabla 2: Variables ítems del cuestionario

\begin{tabular}{cl}
\hline Variable & \\
\hline X42 & Favorecen el desarrollo de enfermedades \\
X43 & Ayudan al crecimiento de ciertas cualidades del individuo como la orientación espacial \\
X44 & Permiten una mayor interacción con individuos con problemas sociales \\
X45 & Impiden un desarrollo de la niñez ya que, en vez de jugar con los niños y las niñas de su misma edad, \\
& están jugando a los videojuegos \\
X46 & Ayudan al desarrollo de la memoria \\
X47 & En algunos casos permiten hacer algo de deporte sin tener que salir de casa \\
X49 & Edad \\
X50 & Sexo \\
X51 & Ocupación
\end{tabular}

Nota: Elaboración propia.

Se ha analizado la consistencia interna mediante la medición de la fiabilidad de los constructos; para ello se ha realizado el análisis factorial exploratorio y se ha considerado el alpha de Cronbach (Nunnally y Bernstein, 1994). La escala global obtuvo un alto grado de fiabilidad, con un alpha Cronbach de 0,9218 y presenta una Medida Kaiser-Meyer-Olkin de adecuación de muestreo del 0,862, bastante aceptable.

El tratamiento estadístico de los datos se ha realizado mediante el programa informático IBM SPSS Statistics, Versión 23 y DYANE, Versión 4, Diseño y análisis de encuestas en investigación social y de mercados (Santesmases, 2009).

Para el contraste de la hipótesis se han utilizado tanto el análisisfactorial de correspondencia como el Cluster Analysis para así dar una mayor validez al estudio (Núñez-Barriopedro, 2010). Se ha utilizado el análisis factorial de correspondencias, porque "es una técnica que permite descubrir las asociaciones entre dos conjuntos de variables de una tabla cruzada de valores medios" (Uriel y Aldás, 2005, p. 5); además, para lograr una mejor interpretación, se ha realizado una rotación con el método varimax. Seguidamente, se hace el análisis de grupos (cluster analysis), ya que es una técnica que permite determinar grupos internamente homogéneos, pero distintos entre sí (Johnson, 2003). Concretamente, se ha calculado el algoritmo de HowardHarris, método descendente, es decir, se divide secuencialmente la totalidad de individuos de la muestra en grupos cada vez más pequeños y homogéneos. Con este método se consigue minimizar la varianza intragrupos y maximizar las varianzas intergrupos de las variables consideradas (Núñez-Barriopedro, 2010). 


\section{Resultados de la investigación}

\section{Agrupación de público consumidor en función de los beneficios y los perjuicios de los videojuegos}

En este apartado se pretende dividir en grupos, según las opiniones tanto positivas como negativas que los sujetos consumidores tienen acerca de los videojuegos.

Tabla 3: Grupos consumidores en función de los beneficios y los perjuicios de los videojuegos

\begin{tabular}{|c|c|c|c|c|c|c|c|c|}
\hline & Total muestra & Grupo 1 & Grupo 2 & Grupo 3 & Grupo 4 & Grupo 5 & Grupo 6 & \\
\hline Número: & 400 & 60 & 54 & 71 & 70 & 86 & 59 & \\
\hline Suma cuadrados & $2.510,36$ & 191,67 & 200,44 & 208,25 & 287,6 & 225,99 & 163,12 & \\
\hline Variables: & & & & & & & & ANOVA/F de Snedecor \\
\hline X42 Media: & 1,95 & $3,43+$ & 2,06 & 1,65 & $-1,53$ & 1,63 & 1,69 & \multirow{2}{*}{$F(5,394)=78,0781 \quad(p=0,0000)$} \\
\hline Des.Est.: & 0,91 & 0,62 & 0,73 & 0,63 & 0,73 & 0,65 & 0,46 & \\
\hline X43 Media: & 2,73 & 2,37 & 3,78 & 2,51 & $3,81+$ & $-2,06$ & 2,08 & \multirow{2}{*}{$F(5,394)=80,8659 \quad(p=0,0000)$} \\
\hline Des.Est.: & 1,03 & 0,75 & 0,81 & 0,6 & 0,78 & 0,72 & 0,7 & \\
\hline X44 Media: & 2,25 & 1,85 & 2,57 & 2,31 & $3,30+$ & $-1,69$ & 1,83 & $F(5,394)=38,4111$ \\
\hline Des.Est.: & 1 & 0,81 & 0,78 & 0,78 & 0,99 & 0,69 & 0,85 & $(p=0,0000)$ \\
\hline X45 Media: & 2,84 & 4,05 & 3,57 & 2,21 & $-1,69$ & 2,02 & $4,27+$ & $F(5,394)=183,7760$ \\
\hline Des.Est.: & 1,22 & 0,78 & 0,76 & 0,63 & 0,57 & 0,73 & 0,48 & $(p=0,0000)$ \\
\hline X46 Media: & 2,67 & 2,15 & $3,76+$ & 2,48 & 3,73 & $-2,01$ & 2,1 & $F(5,394)=72,2263$ \\
\hline Des.Est.: & 1,07 & 0,79 & 0,72 & 0,75 & 0,86 & 0,74 & 0,75 & $(p=0,0000)$ \\
\hline X47 Media: & 1,81 & 1,45 & 2 & $2,55+$ & 2,03 & $-1,16$ & 1,76 & $F(5,394)=31,9973$ \\
\hline \multirow[t]{3}{*}{ Des.Est.: } & 0,87 & 0,59 & 0,9 & 0,78 & 0,96 & 0,37 & 0,74 & $(p=0,0000)$ \\
\hline & & $\begin{array}{l}N^{\circ} \text { de } \\
\text { Grupos }\end{array}$ & $\begin{array}{c}\text { Varianza } \\
\text { intragrupos } \\
\text { con N grupos }\end{array}$ & $\begin{array}{c}\text { Varianza } \\
\text { entre grupos } \\
\text { (explicada) }\end{array}$ & $\begin{array}{c}\% \text { de } \\
\text { varianza } \\
\text { explicada }\end{array}$ & & & \\
\hline & & 6 & $1.277,07$ & $1.233,29$ & 49,13 & & & \\
\hline \multicolumn{9}{|c|}{ X42: Favorecen el desarrollo de enfermedades } \\
\hline \multicolumn{9}{|c|}{ X43: Ayudan al crecimiento de ciertas cualidades del individuo como la orientación espacial } \\
\hline \multicolumn{9}{|c|}{ X44: Permiten una mayor interacción con individuos con problemas sociales } \\
\hline \multicolumn{9}{|c|}{ X45: Impiden un desarrollo de los niños y las niñas ya que, en vez de jugar con pares de su misma edad, están jugando a los videojuegos } \\
\hline \multicolumn{9}{|c|}{ X46: Ayudan al desarrollo de la memoria } \\
\hline
\end{tabular}

Nota: Elaboración propia. 
http://doi.org/10.15359/ree.24-2.12

ROR: https://ror.org/01t466c14 Universidad Nacional, Costa Rica

http://www.una.ac.cr/educare

educare@una.cr

El proceso de generación de grupos ha sido secuencial. En la primera partición el programa ha usado la variable "Impiden un desarrollo de la niñez, ya que, en vez de jugar con los niños y las niñas de su misma edad, están jugando a los videojuegos" por ser la variable que tiene mayor varianza. Con esta primera división se consigue reducir la suma de cuadrados en 24,12 $\%$. A medida que aumenta el número de grupos, aumenta la homogeneidad entre ellos $y$, por tanto, la varianza explicada. Con la última partición realizada se consigue una varianza explicada del 49,12\%, siendo 6 el número de grupos, significativamente diferentes entre sí.

En lo referente a la división en grupos, como se puede apreciar en la Tabla 3, vemos que todos los grupos son significativos al $99 \%$ de confianza.

Respecto al significado de cada uno, se podría nombrar al primero como personas detractoras de los beneficios intelectuales de los videojuegos, pues la variable más importante para ellos es favorecen el desarrollo de enfermedades; el segundo grupo es partidario de los beneficios intelectuales, ya que la variable predominante es ayudan al desarrollo de la memoria; el tercer grupo sería aquel que ve en los videojuegos una forma de hacer deporte, puesto que la variable más importante para ellos es en algunos casos permite hacer algo de deporte sin tener que salir de casa.

En el cuarto grupo se engloba a las personas más partidarias de los videojuegos, pues sus variables más importantes son aquellas que aportan beneficios y, por el contrario, las menos impactantes son las que aportan efectos negativos. El quinto grupo es todo lo contrario, constituido por aquellas personas que tienen peores opiniones sobre los videojuegos, por lo cual le dan mayor importancia a los puntos negativos que a los positivos.

Por último, el sexto grupo estaría enfocado principalmente en las desventajas que los videojuegos aportan a la niñez, este es el grupo más precavido y conservador a la hora de permitir a sus hijos e hijas hacer uso de los videojuegos, pues conciben que les ofrecerían más desventajas que ventajas en su crecimiento.

Como resultado del análisis clúster se pueden apreciar diferentes segmentos. Respecto a un enfoque de marketing, una de las posibles acciones a llevar a cabo es enfatizar el esfuerzo en los grupos con opiniones más negativas para intentar cambiar dichas percepciones. Desde otro punto de vista, se ha llevado a cabo un análisis factorial para analizar las percepciones de los grupos consumidores sobre los efectos que los videojuegos provocan, tanto positivos como negativos. Como se puede ver en la Tabla 4, los resultados de este análisis son significativos al $99 \%$ y presenta una medida Kaiser-Meyer-Olkin de adecuación de muestreo del 0,662, bastante aceptable. Asimismo, el porcentaje de varianza explicada es del 55,76\%. 
Tabla 4: Percepciones acerca de los videojuegos

\begin{tabular}{|c|c|c|c|c|c|c|c|c|c|}
\hline \multicolumn{7}{|c|}{ Medida Kaiser-Meyer-Olkin de adecuación de muestreo } & \multicolumn{3}{|c|}{ 662 } \\
\hline \multirow{3}{*}{\multicolumn{4}{|c|}{ Prueba de esfericidad de Bartlett }} & \multicolumn{3}{|c|}{ Aprox. Chi-cuadrado } & \multicolumn{3}{|c|}{313,042} \\
\hline & & & & \multicolumn{3}{|c|}{ gl } & \multicolumn{3}{|c|}{15} \\
\hline & & & & \multicolumn{3}{|c|}{ Sig. } & \multicolumn{3}{|c|}{, 000} \\
\hline \multirow{2}{*}{ Componente } & \multicolumn{3}{|c|}{ Autovalores iniciales } & \multicolumn{3}{|c|}{ Sumas de extracción de cargas al cuadrado } & \multicolumn{3}{|c|}{ Sumas de rotación de cargas al cuadrado } \\
\hline & Total & $\%$ de varianza & $\%$ acumulado & Total & $\%$ de varianza & $\%$ acu-mulado & Total & $\%$ de varianza & $\%$ acumulado \\
\hline 1 & 2,108 & 35,132 & 35,132 & 2,108 & 35,132 & 35,132 & 1,958 & 32,627 & 32,627 \\
\hline 2 & 1,238 & 20,634 & 55,766 & 1,238 & 20,634 & 55,766 & 1,388 & 23,139 & 55,766 \\
\hline 3 & 883 & 14,712 & 70,478 & & & & & & \\
\hline 4 & ,698 & 11,629 & 82,106 & & & & & & \\
\hline 5 & 613 & 10,220 & 92,327 & & & & & & \\
\hline 6 & ,460 & 7,673 & 100,000 & & & & & & \\
\hline \multicolumn{6}{|c|}{ ¿Cómo crees que impactan los videojuegos en la sociedad? } & \multicolumn{2}{|l|}{ Componente 1} & \multicolumn{2}{|c|}{ Componente 2} \\
\hline \multicolumn{6}{|c|}{ Favorecen el desarrollo de enfermedades. } & & & \multicolumn{2}{|c|}{,837 } \\
\hline \multicolumn{6}{|c|}{$\begin{array}{l}\text { Ayudan al crecimiento de ciertas cualidades del individuo, como la } \\
\text { orientación espacial. }\end{array}$} & \multicolumn{2}{|l|}{, 830} & & \\
\hline \multicolumn{6}{|c|}{ Permiten una mayor interacción con individuos con problemas sociales. } & \multicolumn{2}{|l|}{689} & & \\
\hline \multicolumn{6}{|c|}{$\begin{array}{l}\text { Impiden un desarrollo de la niñez ya que, en vez de jugar con niños o niñas } \\
\text { de su misma edad, están jugando a los videojuegos. }\end{array}$} & & & \multicolumn{2}{|c|}{,781 } \\
\hline \multicolumn{6}{|c|}{ Ayudan al desarrollo de la memoria. } & \multicolumn{2}{|l|}{,771 } & & \\
\hline \multicolumn{6}{|c|}{ En algunos casos permite hacer algo de deporte sin tener que salir fuera de casa. } & \multicolumn{2}{|l|}{, 420} & & \\
\hline
\end{tabular}

Nota: Elaboración propia.

La división realizada por el programa ha sido en dos componentes; en la primera, mayoritariamente, las opiniones de quienes ven puntos positivos en los videojuegos, ya que las variables con más cargas factoriales han sido ayudan al crecimiento de ciertas cualidades del individuo, como la orientación espacial, y ayudan al desarrollo de la memoria. Por otra parte, la segunda componente recoge las opiniones negativas, donde las cargas más influyentes han sido favorecen el desarrollo de enfermedades e impiden el desarrollo de los niños y las niñas.

A quienes se engloban dentro de la componente 1, se les asigna el nombre de grupo partidario de los videojuegos y a quienes se engloban dentro de la componente 2 recibe el nombre de grupo antivideojuegos. Los partidarios de los beneficios son aquellos que ven tanto a nivel intelectual como 
http://doi.org/10.15359/ree.24-2.12

ROR: https://ror.org/01t466c14 Universidad Nacional, Costa Rica

http://www.una.ac.cr/educare

educare@una.cr

a nivel social que los videojuegos aportan algo positivo. Sin embargo, los detractores principalmente ven que además de no aportar nada, influyen negativamente en quienes los juegan.

\section{Conclusiones}

La presente investigación ha permitido analizar el impacto de los videojuegos en los grupos consumidores, al estudiar los efectos positivos y negativos que pueden provocar a través de las percepciones de estos grupos.

La hipótesis $\mathrm{H} 1$ plantea que "los grupos consumidores de videojuegos se diferencian de modo significativo en función de las percepciones positivas y negativas que tienen respecto a cómo influyen estos en la educación. Esta se comprueba y se puede aceptar que se distinguen, significativamente, entre los grupos partidarios de los videojuegos y los grupos antivideojuegos.

Si se entra en más detalle, se pueden distinguir 6 grupos consumidores que presentan diferencias significativas entre sí. Está el grupo detractor de los beneficios intelectuales de los videojuegos, el grupo partidario de los beneficios intelectuales, el grupo que ve en los videojuegos una forma de hacer deporte, el grupo partidario de los videojuegos y, por último, se encuentra el grupo más precavido y conservador a la hora de permitir a sus hijos e hijas hacer uso de los videojuegos.

Estos resultados se acercan a la idea de que los videojuegos presentan una carga mayor de beneficios que de efectos negativos.

Como indican otros estudios (Bourgonjon et ál., 2010), los hombres y las mujeres tienen perspectivas diferentes sobre el impacto de los videojuegos en el público consumidor. Ellas tienen el pensamiento de que son más perjudiciales; sin embargo, ellos ven lo contrario, que aportan beneficios a quienes los juegan.

Así mismo, también cabe destacar que el $100 \%$ de la muestra no aprueba dichos pensamientos, pues a la luz de los resultados existe un porcentaje de población que piensa de una manera más conservadora y ven en estos una posible causa de ciertos problemas de salud, sobre todo, en los niños y niñas en crecimiento.

Por otra parte, se considera que una aplicación más activa de los videojuegos al sector educativo tendría un efecto positivo, pues otros estudios (Revuelta y Guerra, 2012; Sedeño, 2010) explican que, mediante la capacidad inmersiva y la solución tanto pasiva como activa de problemas que se producen en los videojuegos, permiten un desarrollo en ciertas skills de la población joven que, de otra manera, llevaría un mayor tiempo.

A raíz de estos pensamientos se han llevado a cabo estudios en varios grupos de control para probar el efecto positivo que los videojuegos tienen en el aprendizaje. En un grupo se usaba el juego a analizar y en el otro un juego placebo. Los resultados de dichas investigaciones tenían 
que ser tomados con precaución, pues no se había tenido sumo cuidado en la separación de los grupos, pudiendo distorsionar los resultados obtenidos (Bourgonjon et ál., 2010). Sin embargo, Kebritchi et ál. (2010) consiguen desarrollar una investigación con resultados medibles. Estudian los resultados académicos de dos grupos de estudiantes, uno de los grupos había hecho uso de un juego de ordenador y el otro no. Obtuvieron mejores notas quienes habían jugado el videojuego (Rodríguez-Hoyos y Gomes, 2013).

Cabe señalar que los grupos consumidores ya no tienen la idea preconcebida de que los juegos ofrecen principalmente unos perjuicios sobre quienes los consumen. Más bien es el caso contrario, pues gracias a las numerosas investigaciones que avalan los beneficios que ofrecen los videojuegos, se están posicionando en la mentalidad de las personas como un bien beneficioso. Así mismo, están desapareciendo los mitos de que los videojuegos son los causantes de la agresividad de los niños y los posibles actos delictivos de adolescentes, puesto que existen investigaciones que demuestran que no existe correlación alguna entre la violencia y delincuencia y el uso de los videojuegos (Ferguson et ál., 2014).

Por otro lado, el estudio aquí descrito supone un acercamiento al sujeto consumidor de videojuegos, no tanto en lo que representa un hobby sino más bien en una posible aplicación en otros ámbitos, como en la educación o en la medicina. Estas aplicaciones irán en progreso gracias a la aparición de nuevas tecnologías tales como la realidad virtual o la mejora de los ya existentes simuladores. Estos primeros resultados ya se pueden vislumbrar, puesto que existen casos donde los videojuegos se han usado para rehabilitaciones. El reciente boom que hoy día se vive en las nuevas tecnologías a pasos agigantados permitirá, en un futuro, poder mejorar la educación en la era del Big Data. Por ejemplo, a través de la enseñanza en espacios 3D virtuales o mediante entornos más dinámicos y entretenidos, donde el estudiantado podrá disfrutar mientras desarrolla sus habilidades (Núñez-Barriopedro y Vílchez, 2017).

Para finalizar, este estudio sirve para constatar el cambio de imagen que han vivido, en estos últimos años, los videojuegos: la población ha pasado de tener una percepción nociva sobre los videojuegos a otra algo más positiva. En futuras líneas de investigación sería interesante poder analizar en profundidad la influencia que ejercen los videojuegos en la sociedad digital, tanto desde un ámbito docente como profesional.

\section{Referencias}

Asociación Española de Videojuegos (AEVI). (2009). Adese presenta su Anuario 2009 y las expectativas de la industria para la campaña 2010. http://www.aevi.org.es/adese-presentasu-anuario-2009-y-las-expectativas-de-la-industria-para-la-campana-2010/

Bourgonjon, J., Valcke, M., Soetaert, R. y Schellens, T. (2010). Students' perceptions about the use of video games in the classroom. Computers \& Education, 54(4), 1145-1156. https://doi. org/10.1016/j.compedu.2009.10.022 
http://doi.org/10.15359/ree.24-2.12

ROR: https://ror.org/01t466c14 Universidad Nacional, Costa Rica

http://www.una.ac.cr/educare

educare@una.cr

Capel Masip, N., Tejada Fernández, J. y Bosco, A. (2017). Los videojuegos como medio de aprendizaje: Un estudio de caso en matemáticas en educación primaria. Pixel-Bit. Revista de Medios y Educación, 51, 133-150. http://dx.doi.org/10.12795/pixelbit.2017.i51.09

Castellón, L. y Jaramillo, Ó. (2012). Educación y videojuegos: Hacia un aprendizaje inmersivo. En C A. Scolari (Ed.), Homo videoludens 2.0. De Pacman a la gamification (pp. 264-281). Universidad Mayor. https://issuu.com/eslibre.com/docs/homo videoludens 2.0 de pacman a I

Cole, H. y Griffiths, M. D. (2007). Social interactions in massively multiplayer online roleplaying gamers. CyberPsychology \& Behavior, 10(4), 575-583. https://doi.org/10.1089/ cpb.2007.9988

Crescenzi Lanna, L. y Grané Oró, M. (2016). An analysis of the interaction design of the best educational apps for children aged zero to eight. Comunicar. Media Education Research Journal, 24(1), 76-85. http://dx.doi.org/10.3916/C46-2016-08

DEV. Asociación Española de Empresas Productoras y Desarrolladoras de Videojuegos y Software de Entretenimiento. (2015). Libro blanco del desarrollo español de videojuegos 2015. http:// goo.gl/XSoJzO

Díez Gutiérrez, E. J. (2007). El género de la violencia en los videojuegos y el papel de la escuela. Revista de Educación, 342, 127-146. https://www.researchgate.net/profile/Enrique Diez Gutierrez/publication/28161201 El genero de la violencia en los videojuegos y el papel de la escuela/links/0f317535794c4060be000000.pdf

Díez Gutiérrez, E. J., Terrón Bañuelos, E. y Rojo Fernández, J. (2001). Videojuegos: Cuando la violencia vende. Cuadernos de Pedagogía, 305, 1-9. http://buleria.unileon.es/bitstream/ handle/10612/3666/videojuegos-cuando-la.pdf?sequence $=1$

Egenfeldt-Nielsen, S., Smith, J. H. y Pajares Tosca, S. (2019). Understanding video games: The essential introduction. Routledge. https://doi.org/10.4324/9780429431791

Etxeberría Balerdi, F. (2008). Videojuegos y educación. Teoría de la Educación: Educación y Cultura en la Sociedad de la Información, 9(3), 11-28. http://redined.mecd.gob.es/xmlui/bitstream/ handle/11162/91630/00820113013570.pdf?isAllowed $=y \&$ sequence $=1$

Ferguson, C. J., Olson, C. K., Kutner, L. A. y Warner, D. E. (2014). Violent video games, catharsis seeking, bullyi multivariate analysis of effects. Crime \& Delinquency, 60(5), 764-784. https:// doi.org/10.1177/0011128710362201 
http://doi.org/10.15359/ree.24-2.12

ROR: https://ror.org/01t466c14 Universidad Nacional, Costa Rica http://www.una.ac.cr/educare educare@una.cr

González, J.L., Cabrera, M.J.yGutiérrez, F.L. (2007).Diseño de videojuegos aplicados a la educación especial. En J. A. Macías, A. Granollers, P. M. Latorre (Coords.), Actas del VIII Congreso Internacional de Interacción Persona Ordenador (Interacción) (pp. 35-44). Saragoza. https:// www.researchgate.net/profile/Marcelino Cabrera/publication/228544527 Diseno de videojuegos aplicados a la Educacion Especial/links/0fcfd5075b0500ef0d000000/ Diseno-de-videojuegos-aplicados-a-la-Educacion-special.pdf

González-Vázquez, A. e Igartua Perosanz, J. J. (2020). Deshumanización y legitimación de los conflictos armados en los videojuegos bélicos. Palabra Clave, 23(1), e2314. https://doi. org/10.5294/pacla.2019.23.1.4

Gramigna, A. y González-Faraco, J. C. (2009). Videojugando se aprende: Renovar la teoría del conocimiento y la educación. Comunicar, 17(33), 157-164. https://doi.org/10.3916/c332009-03-007

Greenfield, P. M. y Cocking, R. R. (1996). Interacting with video. Ablex Publishing Corporation. $\quad$ https://books.google.es/books?id=QZjJ9ulj1CcC\&pg=PA65\&lpg=PA65 $\& d q=$ Interacting + with+video.+Norwood.+New+Jersey \&source=bl\&ots=eV5lavg $3 b$ I\&sig= uOTRySC5H6NT1EgbNOBhZR9fuY\&hl=es-419\&sa=X\&ved=2ahUKEwjx7Ym06zeAhVsCsAKHWfuCbEQ6AEwCnoECAMQAQ\#v=onepage\&q=Interacting\%20with\%20 video.\%20Norwood.\%20New\%20Jersey\&f=false

Griffiths, M. (2002). The educational benefits of videogames. Education and Health, 20(3), 47-51. http://irep.ntu.ac.uk/id/eprint/15272/1/187769 5405\%20Griffiths\%20Publisher.pdf

Gros, B. (2000). La dimensión socioeducativa de los videojuegos. Edutec. Revista Electrónica de Tecnología Educativa, 12, 1-11. https://doi.org/10.21556/edutec.2000.12.557

Halbrook, Y. J., O'Donnell, A. T. y Msetfi, R. M. (2019). When and how video games can be good: A review of the positive effects of video games on well-being. Perspectives on Psychological Science, 14(6), 1096-1104. https://doi.org/10.1177/1745691619863807

Hernández, J. F. y Cano, Á. P. (2016). La transmisión de valores y responsabilidad social a partir de los videojuegos. Sphera Publica, 1(16), 114-131. http://sphera.ucam.edu/index.php/ sphera-01/article/view/267

Interactive Advertising Bureau Spain (IAB). (2017). Estudio anual de redes sociales. https:// iabspain.es/wp-content/uploads/iab estudioredessociales 2017 vreducida.pdf

Interactive Software Federation of Europe (ISFE). (2019). Key Facts 2019. 2018 trends \& data. https://www.isfe.eu/isfe-key-facts/

Jarvin, L. (2015). Edutainment, games, and the future of education in a digital world. New Directions for Child and Adolescent Development, 147, 33-40. https://doi.org/10.1002/ cad.20082 
http://doi.org/10.15359/ree.24-2.12

ROR: https://ror.org/01t466c14 Universidad Nacional, Costa Rica

http://www.una.ac.cr/educare

educare@una.cr

Johnson, D. E. (2003). Métodos multivariados aplicados al análisis de datos. Thomson.

Kebritchi, M., Hirumi, A. y Bai, H. (2010). The effects of modern mathematics computer games on mathematics achievement and class motivation. Computers y Education, 55(2), 427-443. https://doi.org/10.1016/j.compedu.2010.02.007

Kühn, S., Kugler, D. T., Schmalen, K., Weichenberger, M., Witt, C. y Gallinat, J. (2019). Does playing violent video games cause aggression? A longitudinal intervention study. Molecular Psychiatry, 24(8), 1220-1234. https://doi.org/10.1038/s41380-018-0031-7

López Gómez, S. y Rodríguez Rodríguez, J. (2016). Experiencias didácticas con videojuegos comerciales en las aulas españolas. Didáctica, innovación y multimedia, 33,1-8. https://ddd. uab.cat/record/148417

Mainer, B. (2006). El videojuego como material educativo: La Odisea. ICONO14, 4(1), 48-76. https://doi.org/10.7195/ri14.v4i1.397

Mañas Pérez, A. y Roig-Vila, R. (2019). Las tecnologías de la información y la comunicación en el ámbito educativo. Un tándem necesario en el contexto de la sociedad actual. Revista Internacional d'Humanitats, 45,75-86. http://rua.ua.es/dspace/handle/10045/82089

Marín Díaz, V. y García, Fernández M. D. (2005). Los videojuegos y su capacidad didácticoformativa. Pixel-Bit: Revista de medios y educación, 26, 113-119. http://www.redalyc.org/ pdf/368/36802609.pdf

Marquès, P. (2000). Las claves del éxito. Cuadernos de Pedagogía, 291, 55-58. https://ddd.uab.cat/ record/164400

Martínez, J. (2019). Percepciones de estudiantes y profesores acerca de las competencias que desarrollan los videojuegos. Pensamiento Educativo, 56(2), 1-21. https:// pensamientoeducativo.uc.cl/index.php/pel/article/view/1165/2124

Montes González, J. A., Ochoa Angrino, S., Baldeón Padilla, D. S. y Bonilla Sáenz, M. (2018). Videojuegos educativos y pensamiento científico: Análisis a partir de los componentes cognitivos, metacognitivos y motivacionales. Educación y Educadores, 21(3), 388-408. https://doi.org/10.5294/edu.2018.21.3.2

Nunnally, J. C. y Bernstein, I. H. (1994). Psychometric theory (3a ed.). New York: McGraw-Hill.

Núñez-Barriopedro, E. (2010). Los incentivos que motivan a los jóvenes a estudiar formación profesional. Cómo mejorar su horizonte educativo con el marketing. Prisma Social, 4, 1-34. https://dialnet.unirioja.es/servlet/articulo?codigo $=3632618$ 
Núñez-Barriopedro, E., Monclúz, I. M., y Ravina-Ripoll, R. (2019). El impacto de la utilización de la modalidad B-Learning en la educación superior. Alteridad. Revista de Educación, 14(1), 2639. https://doi.org/10.17163/alt.v14n1.2019.02

Núñez-Barriopedro, E. y Vílchez, E. M. (2017). Estrategias de trabajo colaborativo utilizando twitter y wikispaces. Innovaciones aplicadas. Editorial Académica Española.

Pérez, F. J. (2011). Presente y futuro de la tecnología de la realidad virtual. Revista Creatividad y Sociedad, 16, 1-39. http://www.creatividadysociedad.com/articulos/16/4-Realidad\%20 Virtual.pdf

Pinel Martínez, C., Zurita Ortega, F., Espejo Garcés, T., Chacón Cuberos, R., Castro Sánchez, M. y Pérez Cortés, A. (2016). La interacción de la frecuencia de juego con la adquisición de hábitos saludables en escolares de Granada. RELATEC Revista Latinoamericana de Tecnología Educativa, 15(2),169-176. https://doi.org/10.17398/1695-288X.15.2.169

Revuelta, F. I. y Guerra, J. (2012). ¿Qué aprendo con videojuegos? RED. Una perspectiva de metaaprendizaje del videojugador. Revista de la Educación a Distancia, 33, 1-25. https:// www.um.es/ead/red/33/revuelta.pdf

Ricoy, C. y Ameneiros, A. (2016). Preferencias, dedicación y problemáticas generadas por los videojuegos: Una perspectiva de género. Revista Complutense de Educación, 27(3), 12911308. https://doi.org/10.5209/rev RCED.2016.v27.n3.48445

Rodríguez-Hoyos, C. y Gomes, M. J. (2013). Videojuegos y educación: Una visión panorámica de las investigaciones desarrolladas a nivel internacional. Profesorado. Revista de Currículum y Formación de Profesorado, 17(2), 479-494. http://www.redalyc.org/pdf/567/56729526027.pdf

Rojas, V. (2008). Influencia de la televisión y videojuegos en el aprendizaje y conducta infantojuvenil. Revista chilena de pediatría, 79(Supl. 1), 81-85. https://doi.org/10.4067/S0370$\underline{41062008000700012}$

Russoniello, C. V., O'Brien, K. y Parks, J. M. (2009). EEG, HRV and psychological correlates while playing Bejeweled II: A randomized controlled study. En B. K. Wiederhold y G. Riva (Eds.), Annual review of cyber Therapy and telemedicine 2009. Advancetechnologies in the behavioral, social and neurosciences (pp. 189-192). Interactive Media Institute and IOS Press.

Santesmases, M. (2009). DYANE Versión 4. Diseño y análisis de encuestas en investigación social y de mercados. Pirámide.

Sanz-Gómez, Y. (2017). Investigación de mercado sobre los videojuegos: influencias y consecuencias [trabajo fin de grado inédito]. Universidad Carlos III de Madrid, España. 
http://doi.org/10.15359/ree.24-2.12

ROR: https://ror.org/01t466c14 Universidad Nacional, Costa Rica

http://www.una.ac.cr/educare

educare@una.cr

Sedeño, A. M. (2010). Videojuegos como dispositivos culturales: Las competencias espaciales en educación. Comunicar, 17(34), 183-189. https://doi.org/10.3916/C34-2010-03-18

Serna-Rodrigo, R. (2020). Posibilidades de los videojuegos no serios para el aprendizaje formal de la lengua y la literatura: Una propuesta de clasificación. EDMETIC, Revista de Educación Mediática y TIC, 9(1), 104-125. https://doi.org/10.21071/edmetic.v9i1.12245

Téllez Quirós, J. (2019). Percepción de los costarricenses sobre la conexión entre los videojuegos y tiroteos masivos. Revista Estudios, 38, 207-228. https://doi.org/10.15517/re.v0i38.37468

Teng, Z., Nie, Q., Zhu, Z. y Guo, C. (2020). Violent video game exposure and (Cyber) bullying perpetration among Chinese youth: The moderating role of trait aggression and moral identity. Computers in Human Behavior, 104, 106193. https://doi.org/10.1016/j. chb.2019.106193

Uriel, E. y Aldás, J. (2005). Análisis multivariante aplicado. Ediciones Paraninfo.

Valderrama-Ramos, J. A. (2012). Los videojuegos: conectar alumnos para aprender. Sinéctica, 39, 1-15. https://sinectica.iteso.mx/index.php/SINECTICA/issue/view/42 